\title{
Automated detection of COVID-19 from X-ray images using CNN and Android mobile
}

\author{
Kanij Fatema Bushra ${ }^{1}$ ( $\cdot$ Md. Asif Ahamed ${ }^{2,3} \cdot$ Mohiuddin Ahmad $^{4}$
}

Received: 4 January 2021 / Accepted: 25 July 2021 / Published online: 12 August 2021

(c) Sociedade Brasileira de Engenharia Biomedica 2021

\begin{abstract}
Purpose The prevalence of the coronavirus disease 2019 (COVID-19) pandemic has made a huge impact on global health and the world economy. Easy detection of COVID-19 through any technological tool like a mobile phone can help a lot. In this research, we focus on detecting COVID-19 from X-ray images on Android mobile with the help of Artificial Intelligence (AI). Methods A convolutional neural network (CNN) model is developed in MATLAB and then converted to the CNN model to TensorFlow Lite (TFLite) model to deploy on Android mobile. An Android application is developed which uses the TFLite model to detect COVID-19 using X-ray images.

Results By employing a 5-fold cross-validation, an average accuracy of $98.65 \%$, sensitivity of $98.49 \%$, specificity of $98.82 \%$, precision of $98.81 \%$, and F1-score of $98.65 \%$ are achieved in COVID-19 detection.

Conclusion With our developed Android application, users can detect COVID-19 from X-ray images on Android mobile, and it will be helpful for the diagnosis of COVID-19.
\end{abstract}

Keywords COVID-19 $\cdot$ X-ray image $\cdot$ Android mobile $\cdot$ CNN

\section{Introduction}

In Wuhan city (Hubei, China) at the end of 2019, many pneumonia cases of obscure cause emerged (Lu et al. 2020). A few days later, the virus by which it is caused was identified and the International Committee on Taxonomy of Viruses (ICTV) named the virus "Severe Acute Respiratory Syndrome Coronavirus 2" (SARS-CoV-2) (Rabi et al. 2020). Coronaviruses are RNA virus of the Coronaviridae subfamily, and there are four main subgroups $(\alpha, \beta, \gamma$, and

Kanij Fatema Bushra

kanijbushra@cuet.ac.bd

1 Department of Biomedical Engineering, Chittagong University of Engineering \& Technology, 4349 Chittagong, Bangladesh

2 Department of Biomedical Engineering, Khulna University of Engineering \& Technology, Khulna 9203, Bangladesh

3 Bangladesh Telecommunications Company Limited, Dhaka 1000, Bangladesh

4 Department of Electrical and Electronic Engineering, Khulna University of Engineering \& Technology, Khulna 9203, Bangladesh

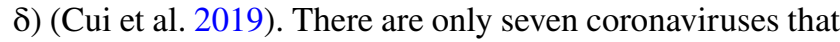
can infect humans, and SARS-CoV-2 is the seventh.

Coronavirus disease (COVID-19) caused by SARSCoV-2 is a highly contagious disease, and the World Health Organization (WHO) declared it a pandemic on $11 \mathrm{March}$ 2020 (Andersen et al. 2020). Reverse transcription polymerase chain reaction (RT-PCR) is a widely used test to detect SARS-CoV-2. However, this test is costly, manual, complex, and consumes time. Other ways to detect COVID-19 are clinical symptoms examination, pathology tests, and radiography. In a meta-analysis, researchers found $87 \%$ sensitivity and $89 \%$ negative predictive value (NPV) in chest computed tomography (CT) compared to RT-PCR and for RT-PCR to get NPV up to $98 \%$; the test should be repeated three times (Khatami et al. 2020). In COVID-19 diagnosis, radiological imaging tools like CT and chest radiograph (CXR) are vital for assessing the evolution of the disease. Those tools are also helpful in finding the stage (mild, moderate, and severe) of the disease. In the early stage of COVID-19, the majority of radiographic images have features like both lung involvement, multi-focal ground-glass opacities (mainly in lower lobes), and in the final stage pulmonary consolidation occurs (Salehi et al. 2020; Huang et al. 2020; Zhang et al. 2020a). In COVID-19 pneumonia, Monaco et al. (2020) preferred 
CXR as a first choice among medical imaging modalities, and in their research, they found substantial interobserver agreement in the CXR severity score.

In automatic diagnosis, machine learning-based solutions have gained popularity, and researchers are using them in many health problems (Waring et al. 2020). Advanced mobile phone technology enables the use of machine learning-based solutions in the mobile phone by which many tasks like face detection (Zhang et al. 2020b), object detection (Zainab 2018), and malware detection (Kim et al. 2019) can be done in the mobile phone. In this research, we developed a CNN model and an Android application to detect COVID-19 from X-ray images on Android mobile.

The contribution of this research is recapitulated below:

1. A novel application is introduced, chest X-ray imagebased COVID-19 detection on Android mobile.

2. Developed a CNN model that can be deployed on Android mobile, works well on Android mobile, and gives very good accuracy.

3. Implemented the $\mathrm{CNN}$ model on Android mobile and developed an Android application to detect COVID-19.

4. A detailed experimental analysis is done in terms of accuracy, sensitivity, specificity, precision, and F1-score.

\section{Related works}

In the COVID-19 epidemic, many Artificial Intelligence (AI) techniques based on medical images (CT and CXR) have been developed to detect COVID-19. There exists both feature extraction-based (Chandra et al. 2021; Mohammed et al. 2020; Elaziz et al. 2020) and deep learning-based approaches to detect COVID-19 from an X-ray image. This review narrates the newly evolved deep learning techniques to detect COVID-19.

Jain et al. (2020) performed transfer learning, and for ResNet101 they got $98.93 \%$ accuracy, $98.66 \%$ specificity, $96.39 \%$ precision, and $98.15 \%$ F-1 score. Their dataset consists of 1215 images which include 250 COVID-19 images. Ucar and Korkmaz (2020) proposed COVIDiagnosis-Net and got $98.26 \%$ accuracy for three classes. Their dataset includes a total of 5949 CXR of which 76 images are of COVID-19. Ozturk et al. (2020) proposed a CNN model named DarkCovidNet and used a total of $1125 \mathrm{X}$-ray images which includes 125 COVID-19 images. In a fivefold crossvalidation for binary classes, an average accuracy of $98.08 \%$ was obtained. El Asnaoui and Chawki (2020) performed transfer learning on seven pre-trained models, and they got the highest accuracy of $92.18 \%$ in Inception-ResNetV2 for three classes. Loey et al. (2020) used a transfer learning technique and three pre-trained models to detect COVID19. They used $306 \mathrm{X}$-ray images which included 69 images of COVID-19 and got an accuracy of $85.20 \%$ on AlexNet for three classes. Apostolopoulos and Mpesiana (2020) used a transfer learning strategy and five pre-trained models to detect COVID-19. A total of 1427 X-ray images, including 224 images of COVID-19 and tenfold cross-validation, were used. For two classes, they got the highest accuracy of $96.78 \%$ in MobiNet V2. Khan et al. (2020) proposed a CNN model named CoroNet and used a total of $1300 \mathrm{X}$-ray images which include 290 images of COVID-19. For three classes, an accuracy of $95 \%$ was obtained. Abraham and Nair (2020) proposed multi-CNN, a combination of several pre-trained CNNs. They used a total of 950 X-ray images which included 453 images of COVID-19 and obtained $91.16 \%$ accuracy for binary classes. Makris et al. (2020) applied transfer learning on nine pre-trained CNN models and for three classes got the highest accuracy of $95.88 \%$ in VGG16. Heidari et al. (2020) used the transfer learning technique, and for VGG-16 they got $94.5 \%$ accuracy. In the above researches, the authors have developed their datasets from different sources, and data from Cohen et al. (2020) is common in all datasets. Civit-Masot et al. (2020) used the VGG16 based CNN model and got $86 \%$ accuracy for three classes. They used 396 X-ray images in total, including 132 of COVID-19. Mahmud et al. (2020) proposed a CNN model named CovXNet and used a total of 5856 X-ray images which includes 305 images of COVID-19. For binary classes, they got $97.4 \%$ accuracy. Goel et al. (2021) used 900 COVID-19 X-ray images and got $97.78 \%$ accuracy in their proposed CNN model named OptCoNet.

\section{Research methodology}

Because CNN models are self-learning, they can achieve high accuracy in image classification while also performing well in multiclass problems. In this paper, inspired by GoogleNet (Szegedy et al. 2015), a CNN model is developed. Though there are many COVID-19 patients, the public availability of X-ray images of COVID-19 positive patients is very small and sporadic. For this, data from multiple sources are used to develop the CNN model. To deploy the model in the Android mobile, the CNN model is converted to the TensorFlow Lite (TFLite) model. An Android application is developed to perform classification on Android mobile.

\section{Database description}

In this research, X-ray images from different sources are used to create one database. This database includes 592 COVID-19 positive X-ray images and 592 normal X-ray images of posterior-anterior (PA) view. Firstly, COVID-19 positive X-ray images hosted on GitHub shared by Cohen et al. (2020) were chosen. Secondly, COVID-19 positive 
$\mathrm{X}$-ray images shared by a physician were chosen (Chest Imaging 2020). Thirdly, normal X-ray images shared by Kermany et al. (2018) were chosen. CXR images were resized to $300 \times 300$. There was no filter used during the resizing, and the aspect ratio was kept at 1:1. Our database includes two classes, and Fig. 1 shows the randomly selected images from class samples. In Fig. 1a, peripheral opacities exist in both lungs.

\section{Proposed CNN model}

Deep learning is a robust scheme to unravel different recognition problems, and it focuses on learning excellent features automatically. Among various deep learning methods, CNN provides very outstanding results in different classification problems. In this research, a 150-layer CNN model is developed to classify, and it is shown in Fig. 2 a. The image input dimension of this network is $300 \times 300 \times 3$. The main reason for selecting this architecture is given below:

- Multilevel convolution filters are applied to the same input, so it ensures multilevel feature extraction.

- Before every convolution filter, $1 \times 1$ convolution is performed, where cross-channel correlation is performed, and it also ignores spatial dimension.

- By performing $1 \times 1$ convolution before every convolution filter, it reduces computational cost largely.

- Through the max-pooling operation, low-level features can be captured.

Our proposed architecture differs from GoogleNet such that instead of several inception modules used in GoogleNet, we used four modified inception modules (shown in Fig. 2b) in our network. Every modified inception module consists of $3 \times 3,5 \times 5,7 \times 7,8 \times 8,21 \times 21,22 \times 22$, and $25 \times 25$ convolution filters, whereas in GoogleNet, only $3 \times 3$ and $5 \times 5$ convolution filters are used in the inception module. In our observation, we found that those seven filters have a good impact on performance and accuracy. The total number of parameters in this model is 9.7 million.

\section{Android application development}

We used MATLAB R2020a to train our proposed CNN model. The trained CNN model is converted to the TFLite model, and Fig. 3d illustrates this conversion process. Firstly, in MATLAB, directed acyclic graph (DAG) network is converted to the open neural network exchange (ONNX) model format. Then the ONNX model is converted to a frozen graph. After that, the freeze graph is converted to the TFLite model. By using the TFLite model, an Android application is developed to classify the X-ray images. This Android application requires a minimum of Android version 6 (API 23) to work. To develop this Android application, Android studio 3.4.2 and TFLite framework are used. To load and execute the CNN model on Android mobile, org. tensorflow.lite package is used. At first, the Android application loads the CXR image and then resizes the image to a $300 \times 300$ size. Then the image is passed through the TFLite model and finally gives classification results.

Figure 4 (b) illustrates the steps for COVID-19 detection on Android mobile. At first, the user will complete a chest X-ray test in a hospital. Then after disinfecting the mobile, the user will take a picture of the X-ray film. Finally, COVID-19 can be detected using that image in the Android application. By this, the user will know the test result before the X-ray report and get some time to take the necessary steps. If a radiologist is unavailable or takes too much time
Fig. 1 X-ray image: a COVID19 , b normal (a)

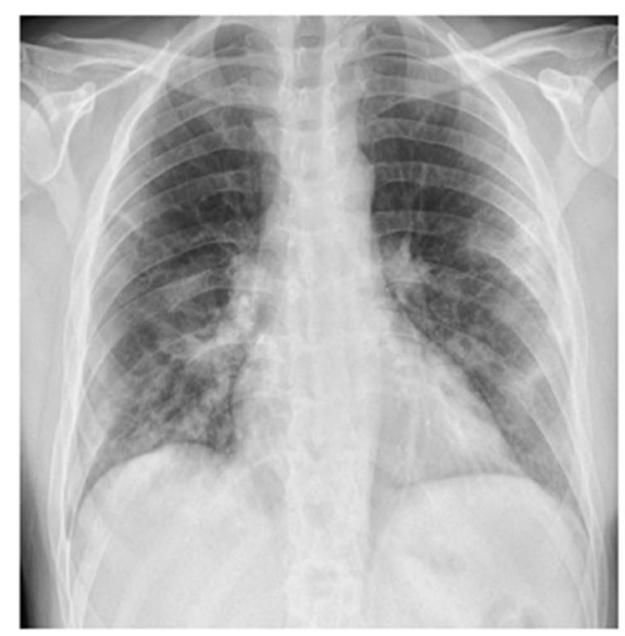

(b)

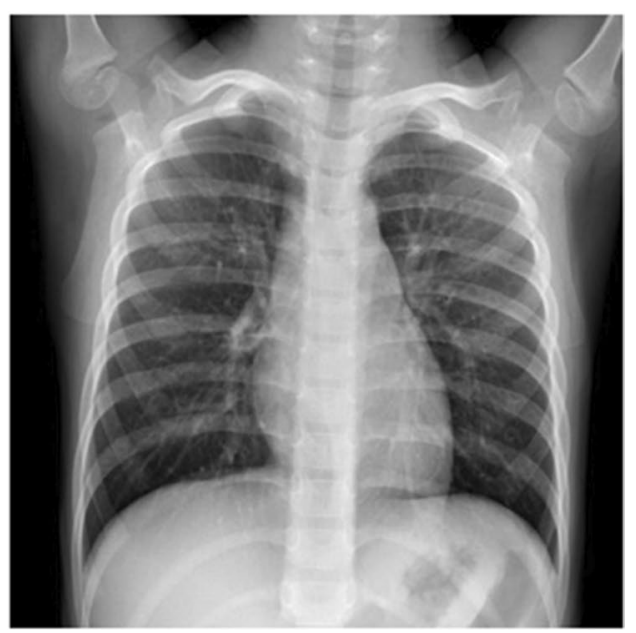


(a)

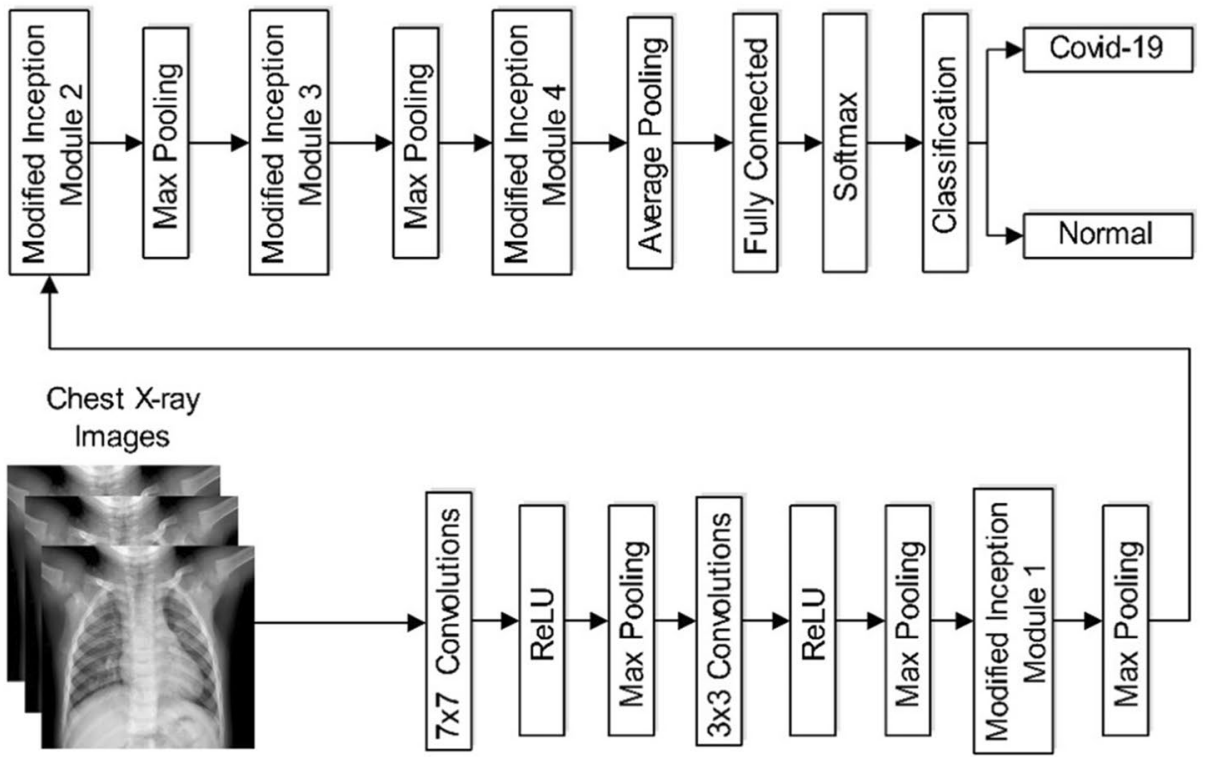

(b)

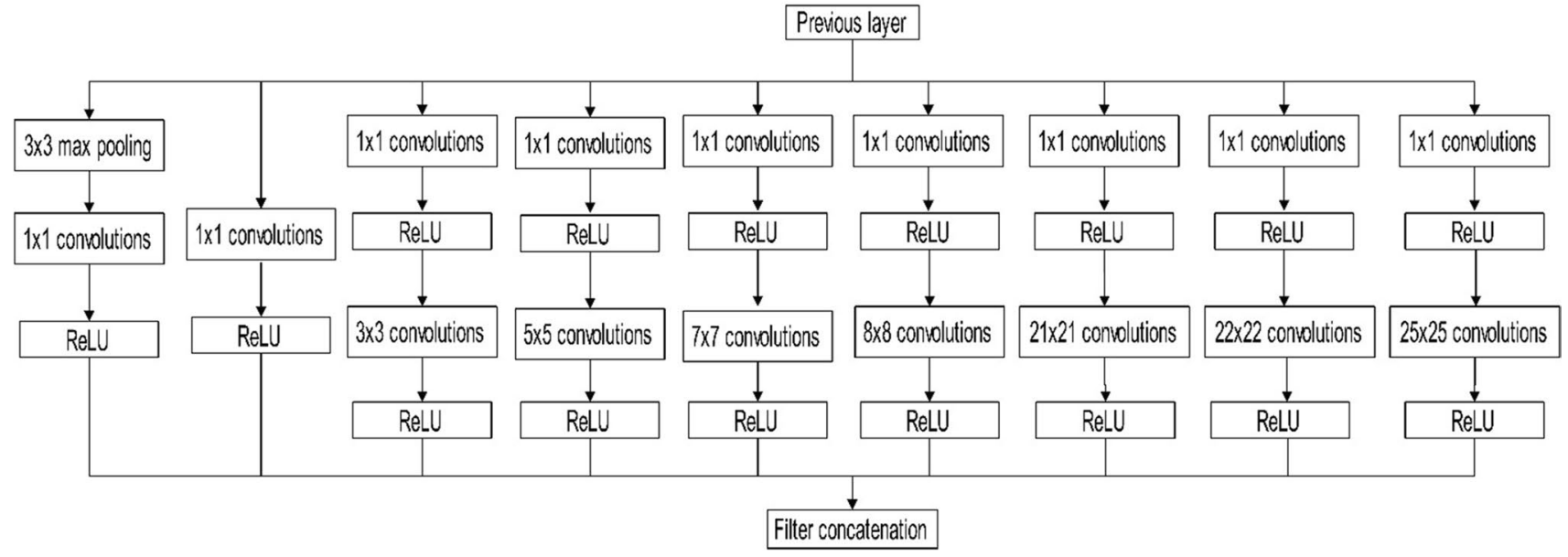

Fig. 2 a Architecture of the proposed CNN model and $\mathbf{b}$ modified inception module used in the CNN model

to verify the X-ray film, the user can verify the X-ray film with this Android application.

A screenshot of the Android application is shown in Fig. 3 a, b, and c. To use this Android application, firstly, the user will open an X-ray image with the OPEN PICTURE button, and after that, the user needs to press the CLASSIFY button (shown in Fig. 3 a). For the first time, this application will request permission to access photos and media. Then by pressing the CLASSIFY NOW button, the probability of the two classes will be displayed (shown in Fig. $3 \mathrm{~b}$ and c). Among the two classes which belong to higher probability, X-ray image will belong to that class.
Figure $3 \mathrm{~b}$ and $\mathrm{c}$ show the classification result for COVID19 and normal X-ray image.

\section{Experimental results}

The experimental appraisement of our proposed model was done on MATLAB R2020a. For this, a computer having an AMD Ryzen 3700X processor, 32 GB RAM, and NVIDIA GTX 10606 GB Graphics Processing Unit (GPU) is used. Our proposed model was trained using Stochastic Gradient Descent (SGD) with momentum optimizer having 0.001 learning rate, 
(a)

\begin{tabular}{ll}
$12: 23$ & ङ $A 4,40$ \\
$M L$ & \\
\hline
\end{tabular}

$$
\begin{aligned}
& \text { OPEN PICTURE } \\
& \text { CLASSIFY }
\end{aligned}
$$

(b)
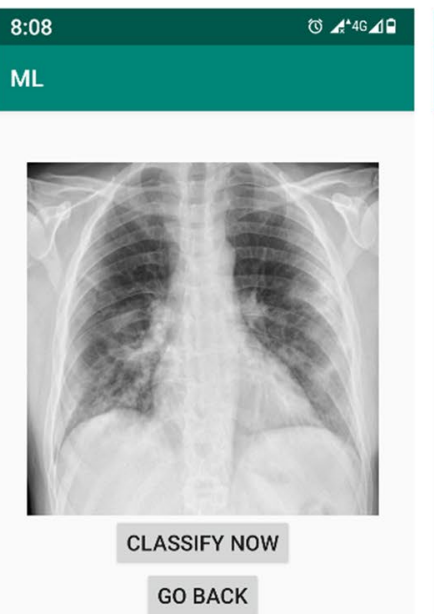

Probability

1. Covid-19

2. Normal (c)
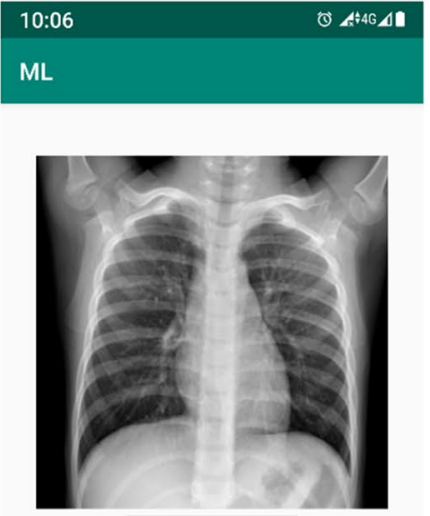

CLASSIFY NOW

GO BACK

Probability

1. Normal

2. Covid-19 (d)

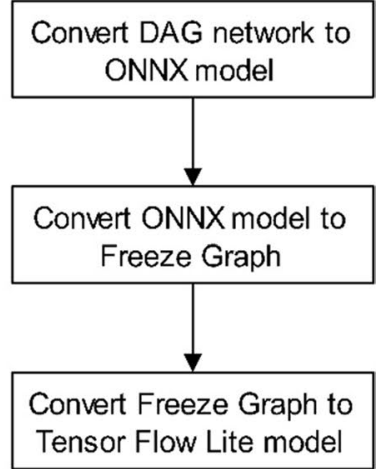

Fig. 3 a,b,c Developed Android application and $\mathbf{d}$ TFLite model conversion

0.9 momentum, and 175 mini-batch sizes with 150 epochs. To evaluate the performance of the proposed model, fivefold cross-validation is employed. The confusion matrix for each fold is shown in Fig. 4a. To appraise the performances of the proposed model, accuracy, sensitivity, specificity, precision, and F1-score are used, and they are shown in Table 1.

\section{Discussion}

In this research, we proposed a $\mathrm{CNN}$ model and developed an Android application by using that CNN model to detect COVID-19 from X-ray images on Android mobile. We employed fivefold cross-validation and achieved $98.65 \%$ average accuracy. There exist many deep learning techniques to detect COVID-19 from X-ray images and a comparison between them and our proposed method is given in
Fig. 4 a Confusion matrix and b steps for COVID-19 detection on Android mobile (a)

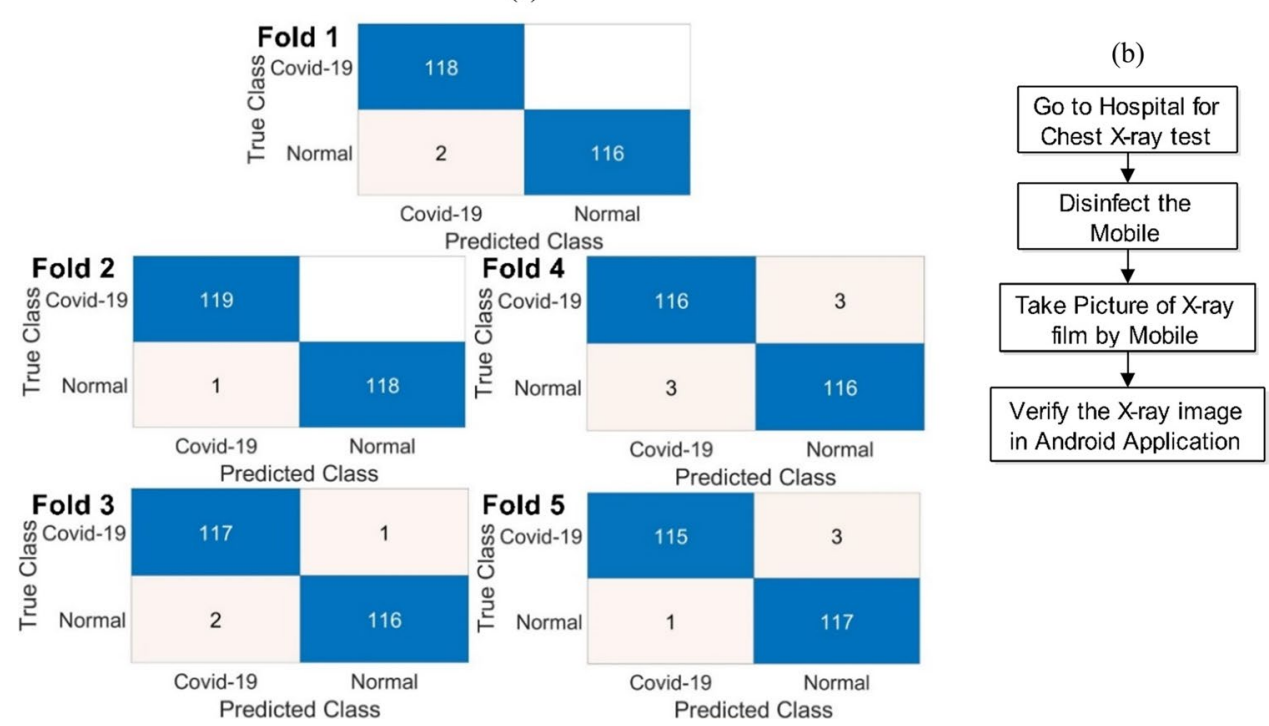


Table 1 Performance matrices for each fold

\begin{tabular}{llllll}
\hline Fold & \multicolumn{5}{l}{ Performance matrices $(\%)$} \\
\cline { 2 - 6 } & Accuracy & Sensitivity & Specificity & Precision & F1-score \\
\hline Fold-1 & 99.15 & 98.34 & 100 & 100 & 99.16 \\
Fold-2 & 99.58 & 99.17 & 100 & 100 & 99.58 \\
Fold-3 & 98.73 & 98.32 & 99.14 & 99.15 & 98.73 \\
Fold-4 & 97.48 & 97.48 & 97.48 & 97.48 & 97.48 \\
Fold-5 & 98.31 & 99.13 & 97.50 & 97.46 & 98.29 \\
Average & 98.65 & 98.49 & 98.82 & 98.81 & 98.65
\end{tabular}

Table 2. Many researchers have employed transfer learning techniques, and from Table 2, the highest accuracy was obtained in ResNet101 for transfer learning. It has slightly higher accuracy than our proposed model, but in terms of specificity, precision, and F-1 score, it lags. Ucar and Korkmaz (2020); Ozturk et al. (2020); Khan et al. (2020); Abraham and Nair (2020); Mahmud et al. (2020); and Goel et al. (2021) have proposed their own CNN model, and among them, Ucar and Korkmaz (2020) got the highest accuracy of 98.26 which lags our one. The dataset used by Goel et al. (2021) includes the same two data sources which we used in this research. They got $97.78 \%$ accuracy, which lags our one.

We have developed our proposed CNN model in such a way that it works well on Android mobile and compatible with the TFLite built-in operator library so that CNN model deployment on Android mobile is possible with a very good accuracy. In Table 2, only our proposed method is implemented in real-life scenarios like Android mobile, and it makes this research unique from others.

\section{Conclusion}

As COVID-19 is highly contagious, it is essential to detect every positive case very fast. This will significantly reduce viral transmission. Deep learning and mobile phones can play a vital role in this case. With the advancement in mobile phone technology, it has become possible to deploy pretrained models on Android mobile for classification. For this, we focused on COVID-19 detection on Android mobile from X-ray images and implemented it on Android mobile. We obtained an average accuracy of $98.65 \%$, a sensitivity of $98.49 \%$, a specificity of $98.82 \%$, a precision of 98.81 , and an F1-score of $98.65 \%$ for our proposed CNN model. After deploying this model on Android mobile, our developed Android application successfully predicts COVID-19 and normal X-ray images. With our developed Android application, after taking pictures of X-ray film from a mobile phone camera, the user can verify their X-ray images on their Android mobile. The user must avoid self-diagnosis, and before starting diagnosis, the user must verify the X-ray film by a radiologist. We hope that the $\mathrm{CNN}$ model and Android application in our proposed research will be helpful for the diagnosis of COVID-19.

Our proposed work has some limitations. Firstly, our developed Android application will not work below Android version 6 (API 23), and due to this, the user's old Android mobile will not be able to run it. Secondly, in this research, we used only X-ray images of PA view, and for this our proposed CNN model will not work on $\mathrm{X}$-ray images in other views like lateral and anterior-posterior (AP). Thirdly, stages (mild, moderate, and severe) of pneumonia due to COVID-19 cannot be detected. Fourthly, we used a limited number of COVID-19 X-ray images due to the scarcity of COVID-19 CXR data. Our
Table 2 Comparison between the proposed method and existing methods

\begin{tabular}{llll}
\hline Author & Year & Architecture & Accuracy (\%) \\
\hline Jain et al. (2020) & 2020 & ResNet101 & 98.93 \\
Ucar and Korkmaz (2020) & 2020 & COVIDiagnosis-Net & 98.26 \\
Ozturk et al. (2020) & 2020 & DarkCovidNet & 98.08 \\
El Asnaoui and Chawki (2020) & 2020 & Inception-ResNetV2 & 92.18 \\
Loey et al. (2020) & 2020 & AlexNet & 85.20 \\
Apostolopoulos and Mpesiana (2020) & 2020 & MobileNet V2 & 96.78 \\
Khan et al. (2020) & 2020 & CoroNet & 95.00 \\
Abraham and Nair (2020) & 2020 & Multi-CNN & 91.16 \\
Makris et al. (2020) & 2020 & VGG16 & 95.88 \\
Heidari et al. (2020) & 2020 & VGG16 & 94.50 \\
Civit-Masot et al. (2020) & 2020 & VGG16 & 86.00 \\
Mahmud et al. (2020) & 2020 & CovXNet & 97.40 \\
Goel et al. (2021) & 2021 & OptCoNet & 97.78 \\
Proposed method & 2021 & CNN & 98.65 \\
\hline
\end{tabular}


proposed CNN model works very well and gives very good accuracy within our dataset. By our developed Android application, we have also tested several X-ray images that are not included in our dataset, and it gives a satisfactory result. But its accuracy may change in mass level implementation, and for proper real-life implementation at a mass level, more COVID-19 CXR is needed. In the future, we will overcome those limitations by extending our work with more COVID-19 X-ray images in different views (PA/AP/lateral).

\section{Declarations}

Conflict of interest The authors declare no competing interests.

\section{References}

Abraham B, Nair MS. Computer-aided detection of COVID-19 from $\mathrm{X}$-ray images using multi-CNN and Bayesnet classifier. Biocybern Biomed Eng. 2020;40:1436-45. https://doi.org/10.1016/j. bbe.2020.08.005.

Andersen KG, Rambaut A, Lipkin WI, Holmes EC, Garry RF. The proximal origin of SARS-CoV-2. Nat Med. 2020;26:450-2. https://doi.org/10.1038/s41591-020-0820-9.

Apostolopoulos ID, Mpesiana TA. Covid-19: automatic detection from $\mathrm{X}$-ray images utilizing transfer learning with convolutional neural networks. Phys Eng Sci Med. 2020;43:635-40. https://doi.org/10. 1007/s13246-020-00865-4.

Chandra TB, Verma K, Singh BK, Jain D, Netam SS. Coronavirus disease (COVID-19) detection in Chest X-Ray images using majority voting based classifier ensemble. Expert Syst Appl. 2021;165: 113909. https://doi.org/10.1016/j.eswa.2020.113909.

Chest Imaging. This is a thread of COVID-19 CXR (all SARS-CoV-2 PCR+) from my hospital (Spain). 2020. https://twitter.com/Chest Imaging/status/12.

Civit-Masot J, Luna-Perejón F, Domínguez Morales M, Civit A. Deep learning system for COVID-19 diagnosis aid using X-ray pulmonary images. Appl Sci. 2020;10:4640. https://doi.org/10.3390/ app10134640.

Cohen JP, Morrison P, Dao L, Roth K, Duong TQ, Ghassemi M. COVID-19 Image data collection: prospective predictions are the future. 2020. https://arxiv.org/abs/2006.11988. Accessed 8 Oct 2020.

Cui J, Li F, Shi Z-L. Origin and evolution of pathogenic coronaviruses. Nat Rev Microbiol. 2019;17:181-92. https://doi.org/10. 1038/s41579-018-0118-9.

El Asnaoui K, Chawki Y. Using X-ray images and deep learning for automated detection of coronavirus disease. J Biomol Struct Dyn. 2020. https://doi.org/10.1080/07391102.2020.1767212.

Elaziz MA, Hosny KM, Salah A, Darwish MM, Lu S, Sahlol AT. New machine learning method for image-based diagnosis of COVID19. PLoS ONE. 2020;15: e0235187. https://doi.org/10.1371/journ al.pone.

Goel T, Murugan R, Mirjalili S, Chakrabartty DK. OptCoNet: an optimized convolutional neural network for an automatic diagnosis of COVID-19. Appl Intell. 2021;51:1351-66. https://doi.org/10. 1007/s10489-020-01904-z.

Heidari M, Mirniaharikandehei S, Khuzani AZ, Danala G, Qiu Y, Zheng B. Improving the performance of $\mathrm{CNN}$ to predict the likelihood of COVID-19 using chest X-ray images with preprocessing algorithms. Int J Med Informatics. 2020;144: 104284. https://doi.org/10.1016/j.ijmedinf.2020.104284.

Huang C, Wang Y, Li X, Ren L, Zhao J, Hu Y, et al. Clinical features of patients infected with 2019 novel coronavirus in Wuhan. China the Lancet. 2020;395:497-506. https://doi.org/10.1016/S01406736(20)30183-5.

Jain G, Mittal D, Thakur D, Mittal MK. A deep learning approach to detect Covid-19 coronavirus with X-Ray images. Biocybernetics and Biomedical Engineering. 2020;40:1391-405. https://doi.org/ 10.1016/j.bbe.2020.08.008.

Kermany DS, Goldbaum M, Cai W, Valentim CCS, Liang H, Baxter SL, et al. Identifying medical diagnoses and treatable diseases by image-based deep learning. Cell. 2018;172:1122-1131.e9. https:// doi.org/10.1016/j.cell.2018.02.010.

Khan AI, Shah JL, Bhat MM. CoroNet: A deep neural network for detection and diagnosis of COVID-19 from chest x-ray images. Comput Methods Programs Biomed. 2020;196: 105581. https:// doi.org/10.1016/j.cmpb.2020.105581.

Khatami F, Saatchi M, Zadeh SST, Aghamir ZS, Shabestari AN, Reis LO, et al. A meta-analysis of accuracy and sensitivity of chest CT and RT-PCR in COVID-19 diagnosis. Sci Rep. 2020;10:22402. https://doi.org/10.1038/s41598-020-80061-2.

Kim T, Kang B, Rho M, Sezer S, Im EG. A multimodal deep learning method for Android malware detection using various features. IEEE TransInformForensic Secur. 2019;14:773-88. https://doi. org/10.1109/TIFS.2018.2866319.

Loey M, Smarandache F, M. Khalifa NE (2020) Within the lack of chest COVID-19 X-ray dataset: a novel detection model based on GAN and deep transfer learning. Symmetry. 12:651. https://doi. org/10.3390/sym12040651.

$\mathrm{Lu} \mathrm{H}$, Stratton CW, Tang Y. Outbreak of pneumonia of unknown etiology in Wuhan, China: the mystery and the miracle. J Med Virol. 2020;92:401-2. https://doi.org/10.1002/jmv.25678.

Mahmud T, Rahman MA, Fattah SA. CovXNet: a multi-dilation convolutional neural network for automatic COVID-19 and other pneumonia detection from chest X-ray images with transferable multireceptive feature optimization. Comput Biol Med. 2020;122: 103869. https://doi.org/10.1016/j.compbiomed.2020.103869.

Makris A, Kontopoulos I, Tserpes K. COVID-19 detection from chest $\mathrm{X}$-Ray images using deep learning and convolutional neural networks. 11th Hellenic Conference on Artificial Intelligence, Athens Greece: ACM; 2020. https://doi.org/10.1145/3411408.3411416.

Monaco CG, Zaottini F, Schiaffino S, Villa A, Della Pepa G, Carbonaro LA, et al. Chest X-ray severity score in COVID-19 patients on emergency department admission: a two-centre study. Eur Radiol Exp. 2020;4:68. https://doi.org/10.1186/s41747-020-00195-w.

Ozturk T, Talo M, Yildirim EA, Baloglu UB, Yildirim O, Rajendra AU. Automated detection of COVID-19 cases using deep neural networks with X-ray images. Comput Biol Med. 2020;121: 103792. https://doi.org/10.1016/j.compbiomed.2020.103792.

Rabi FA, Al Zoubi MS, Kasasbeh GA, Salameh DM, Al-Nasser AD. SARS-CoV-2 and Coronavirus Disease 2019: What We Know So Far. Pathogens. 2020;9:231. https://doi.org/10.3390/pathogens9 030231.

Salehi S, Abedi A, Balakrishnan S, Gholamrezanezhad A. Coronavirus disease 2019 (COVID-19): a systematic review of imaging findings in 919 patients. Am J Roentgenol. 2020;215:87-93. https:// doi.org/10.2214/AJR.20.23034.

Szegedy C, Wei Liu, Yangqing Jia, Sermanet P, Reed S, Anguelov D, et al. Going deeper with convolutions. 2015 IEEE Conference on Computer Vision and Pattern Recognition (CVPR), Boston, MA, USA: IEEE; 2015. https://doi.org/10.1109/CVPR.2015.7298594.

Ucar F, Korkmaz D. COVIDiagnosis-Net: Deep Bayes-SqueezeNet based diagnosis of the coronavirus disease 2019 (COVID-19) 
from X-ray images. Med Hypotheses. 2020;140: 109761. https:// doi.org/10.1016/j.mehy.2020.109761.

Mohammed S, Alkinani F, Hassan Y. Automatic computer aided diagnostic for COVID-19 based on chest X-ray image and particle swarm intelligence. Int J Intell Eng Syst. 2020;13:63-73. https:// doi.org/10.22266/ijies2020.1031.07.

Waring J, Lindvall C, Umeton R. Automated machine learning: Review of the state-of-the-art and opportunities for healthcare. Artif Intell Med. 2020;104: 101822. https://doi.org/10.1016/j.artmed.2020. 101822.

Zainab A. Realtime Object Detection on Android using Tensorflow. Qatar Foundation Annual Research Conference Proceedings Volume 2018 Issue 3, Qatar National Convention Center (QNCC), Doha, Qatar: Hamad bin Khalifa University Press (HBKU Press); 2018. https://doi.org/10.5339/qfarc.2018.ICTPP417.
Zhang N, Wang L, Deng X, Liang R, Su M, He C, et al. Recent advances in the detection of respiratory virus infection in humans. J Med Virol. 2020a;92:408-17. https://doi.org/10.1002/jmv. 25674.

Zhang X, Cheng D, Jia P, Dai Y, Xu X. An efficient Android-based multimodal biometric authentication system with face and voice. IEEE Access. 2020b;8:102757-72. https://doi.org/10.1109/ ACCESS.2020.2999115.

Publisher's note Springer Nature remains neutral with regard to jurisdictional claims in published maps and institutional affiliations. 